\title{
Influence of Doped Anions on Poly (3,4-ethylenedioxythiophene) as Hole Conductors for Solid-State Dye-Sensitized Solar Cells
}

Jiangbin Xia, $\uparrow$ Naruhiko Masaki, $\uparrow$ Monica Lira-Cantu, $\uparrow$ Yukyeong Kim, $\uparrow$ Kejian Jiang, $\dagger$ and Shozo Yanagida*†

Center for Advanced Science and Innovation, Osaka University, Suita, Osaka 565-0871, Japan, Institut de Ciència de Materials de Barcelona. Campus UAB Bellaterra E-08193, Spain

Supporting information

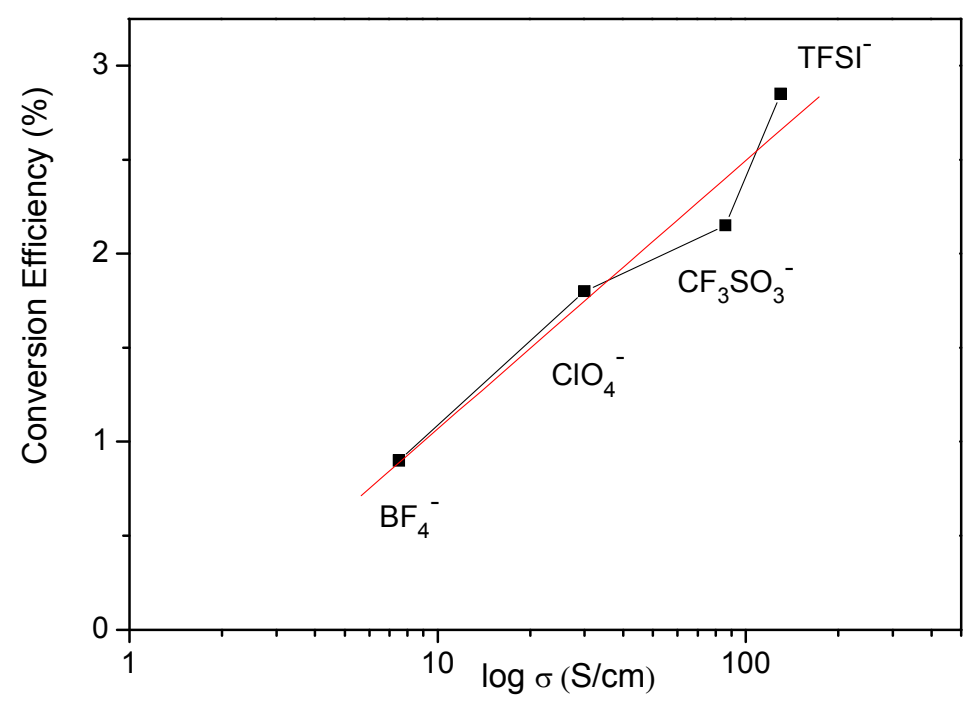

Fig S1. The dependence of conversion efficiency with conductivity of PEDOT hole conductor. 